\title{
Wind Speed Forecasting in China: A Review
}

\author{
Huiru Zhao, Sen Guo* \\ School of Economics and Management, North China Electric Power University, Changping District, Beijing, China
}

\section{Email address:}

guosen324@163.com (Sen Guo), guosen@ncepu.edu.cn (Sen Guo)

\section{To cite this article:}

Huiru Zhao, Sen Guo. Wind Speed Forecasting in China: A Review. Science Journal of Energy Engineering. Special Issue: Soft Computing Techniques for Energy Engineering. Vol. 3, No. 4-1, 2015, pp. 14-21. doi: 10.11648/j.sjee.s.2015030401.13

\begin{abstract}
China's wind power has developed rapidly in the past few years, the large-scale penetration of which will bring big influence on power systems. The wind speed forecasting research is quite important because it can alleviate the negative impacts. This paper reviews the current wind speed forecasting techniques in China. The literature (written in Chinese) sources and classification were firstly analyzed, and then the wind speed forecasting techniques in China were detailed reviewed from four aspects, which are statistical method, soft computing method, hybrid forecasting method and other forecasting methods. This paper can rich the current research in the field of wind speed forecasting.
\end{abstract}

Keywords: Wind Speed Forecasting, Forecasting Techniques, China

\section{Introduction}

In the past few years, the wind energy, as a kind of renewable energy, has experiences a rapid development. At the end of 2013, the installed capacity of wind power in the world has amounted to $318105 \mathrm{MW}$. Of which, the installed wind power capacity of China reached to $91412 \mathrm{MW}$, taking a $28.74 \%$ share of the whole world [1]. In 2010, China's cumulative installed wind power capacity amounted to $41827 \mathrm{MW}$, which surpasses that of USA in term of installed capacity to rank first in the world and has kept the first place till now [2]. It is foreseeable that under the strong support of renewable energy supporting development policies from central government and local governments, the wind power in China will still develop fast in the next few year. It is predicted that the installed wind power capacity in China will increase to 0.2 billion $\mathrm{kW}$ at the end of 2020, and the wind power generation will account for $5 \%$ in the total power generation [3].

Wind power has the intermittent and fluctuant characteristics. Large-scale wind power penetration will bring new challenges to the safe and stable operation of power systems. When the penetration rate of wind power exceeds to a certain value, it will certainly affect the electric power quality and safe operation of power systems. Therefore, accurately forecasting the wind speed and wind power generation play an important role in planning and designing of wind farms, performing unit commitment, keeping the safe operation of power systems and improving the economic and social benefits.

China's wind power develops fast, which accesses to grid will take a huge impact on the power systems. To reduce the side effects, it is quite necessary to forecast the wind speed and wind power generation. In the past few years, Chinese researchers and scholars have performed some studies on forecasting related to wind power, and made some achievements. Currently, there are several papers reviewing the wind power forecasting issues, which focus on the peer-review journal articles written in English [4-6]. However, considering the rapid development of China's wind power and substantial articles written in Chinese which also contribute to the wind power forecasting issue, this paper performs the review on forecasting issue related to wind power in China, namely the current journal articles written in Chinese. The wind power generation will be largely affected by wind speed. When the wind speed is forecasted, the wind power generation can be calculated given the wind turbine type and wind farm location. Moreover, most of the journal articles written in Chinese are related to wind speed forecasting. Therefore, this paper focuses on the wind speed forecasting, which will fill the international review gap in the field of wind speed forecasting.

\section{Literature Sources and Classification}

The purpose of this paper is to review the wind speed forecasting in China. The reviewed literatures are selected from the high quality peer-reviewed journals written in 
Chinese, which are indexed by EI (Engineering Index) and SCI (Science Citation Index) databases.

By indexing the wind speed forecasting in the EI and SCI databases subjected to the peer-reviewed articles written in Chinese, 50 article are finally obtained, which mainly come from Journals “Power System Technology ('电网技术” in Chinese)”, “Acta Energiae Solaris Sinica ('太阳能学报” in Chinese)", "Power System Protection and Control ('电力系统 保护与控制' in Chinese)”, “Proceedings of the CSEE ('中国 电机工程学报” in Chinese)”, and so on. The detailed sourced journals are shown in Figure 1. It can be seen the Power System Technology journal has published the most articles related to wind speed forecasting in the past few years, which account for $24 \%$. The journals Acta Energiae Solaris Sinica, Power System Protection and Control, Proceedings of the CSEE have also published some wind speed forecasting articles, which account for $18 \%, 18 \%$ and $16 \%$, respectively.

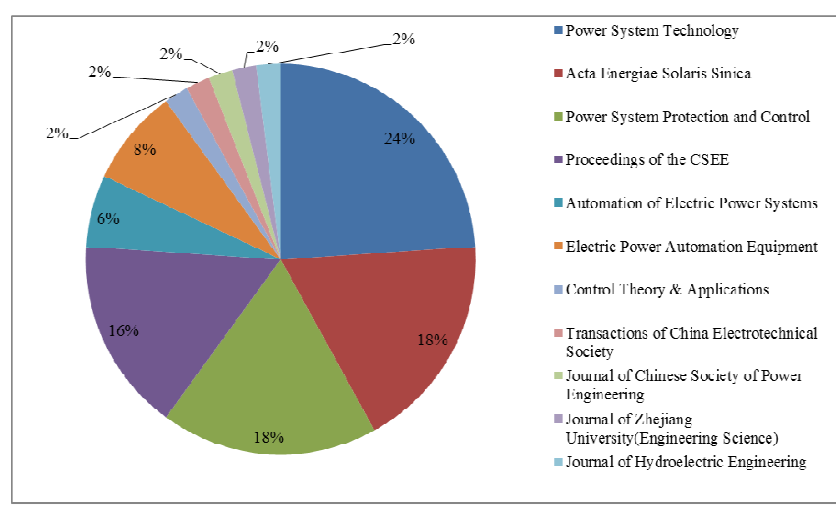

Figure 1. The Chinese high-quality journals publishing wind speed forecasting papers.

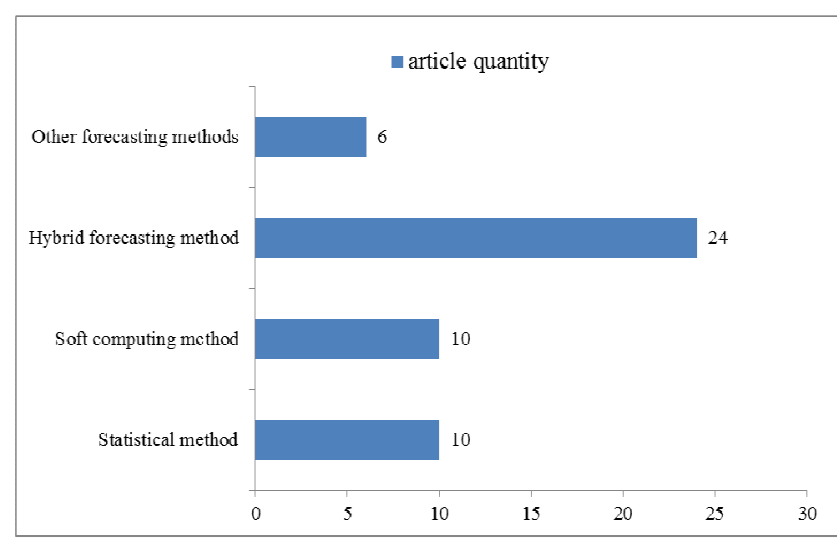

Figure 2. Article distribution sorted by different forecasting techniques.

According to the proposed forecasting techniques of these 50 literatures, four classifications can be obtained, which are statistical method, soft computing method, hybrid forecasting method and other forecasting methods. Different articles employing different wind speed forecasting techniques will be reviewed in detail in the following Sections 3-6, and the distribution is shown in Figure 2. It can be seen the articles employing the hybrid computing method are most, accounting for $48 \%$; the following is statistical methods $(20 \%)$ and soft computing method $(20 \%)$. Therefore, we can safely say that the hybrid forecasting technique for wind speed is dominated in China. There are also some researchers employ the statistical forecasting technique and soft computing forecasting technique to forecast wind speed. This also implies that the hybrid wind speed forecasting method which combines different methods may has a better forecasting performance in the field of wind speed forecasting.

\section{Statistical Method}

The statistical approach is based on training with measurement data and uses difference between the predicted and the actual wind speeds in immediate past to tune model parameters.

Ref. [7] considered the wind speed serial has the characteristics of sequence and autocorrelation, and then forecasted the wind speed based on the time serial analysis technique. Meanwhile, AIC (An Information Criterion) was adopted to verify the effectiveness of this wind speed forecasting model. Ref. [8] proposed a hybrid algorithm integrating time series analysis with Kalman filter to forecast wind speed, and the case study results show the forecasting accuracy of this proposed model can be improved and the time delay in the forecasting can also be well solved. Ref. [9] combined the Wavelet transform theory and time series analysis technique to forecast the wind speed, and the result shows the wind speed is firstly preceded by Wavelet transform theory can improve the forecasting accuracy. Ref. [10] presented a short-term wind speed simulation model based on Kaimal's wind speed power spectrum. The simulated wind speed series were compared to field measured wind speed time series. The results show good performance for simulation collectivity from the model, while it failed to reflect the specific wind speed fluctuation characteristic related to the simulated site. Hence, this paper proposed a method that employs the field measured wind speed to correct the simulated wind speed. After correcting, the simulated wind speed series not only possessed good performance for simulation collectivity, but reflected excellently the specific site characteristic wind speed fluctuation as well. The proposed method can enormously improve the performance of the model.

Ref. [11] used the wavelet decomposition method to decompose the wind speed into several different frequency bands, and then employed the different recursive least square (RLS) models to forecast each band, finally these forecasting results of high frequency bands and low frequency bands were combined to obtain the final forecasting results. The simulation experiment shows the average value of the mean absolute percentage error (MAPE) of wind speed forecasting is $12.25 \%$. Ref. [12] used the method named as vectorization of univariate hourly wind speed time series for eliminating diurnal non-stationary, and vectorized hourly wind speed was expressed as a vector auto regression (VAR) model to forecast hourly wind speed. The results showed that the presented VAR model can yield satisfactory hourly wind speed forecast as 
long as $72 \mathrm{~h}$ ahead under normal weather conditions. Ref. [13] used the ARIMA (autoregressive integrated moving average) technique to forecast the wind speed, and the identified coefficient and AIC (Akaike information criterion) were employed to deal with to establish the ARIMA model. The forecasting result on the next one day's wind speed shows this method is effective. Ref. [14] employed the statistical clustering analysis technique to forecast the wind speed. By processing the sample data using maximal similarity criteria of statistical clustering approach, the prediction of wind speed based on ARIMA model was performed. The prediction accuracy of this proposed method is improved compared to the conventional ARIMA process. Based on study of long-term wind speed prediction using time series model, a new prediction scheme is proposed in Ref. [15]. The wind speed signal was firstly decomposed into trend signal and no-trend random signal, then the latter on was processed and compared respectively using sliding filter method and wavelet analysis method, and finally both the trend signal and previously processed signal were separately predicted and superposed so as to forecast the wind speed. Results show that both the sliding filter method and wavelet analysis method can realize long-term wind speed prediction with a high precision. A novel multi-step prediction for wind speed based on empirical mode decomposition (EMD) is presented in Ref. [16]. By means of the EMD technique, the original wind speed sequences were firstly decomposed into a series of functions with more stationary variation. Thus the interferences among the characteristic information embedded in the wind speed can be weakened. Then these functions were reconstructed into three components (high-middle-low frequency components) according to their run-lengths. After that, three multi-step prediction models were built on the basis of their respective variation rules. Finally, the prediction values corresponded to the three components were adaptively superposed to obtain the predicted wind speed. The forecasting results show that the proposed approach possesses higher accuracy and the prediction performance is satisfied when the wind speed sharply fluctuates.

\section{Soft Computing Method}

The soft computing method for wind speed forecasting refers to employing the machine learn technique or artificial intelligence technique to forecast the wind speed, such as neural network, support vector machine, and so on.

Ref. [17] proposed a wind speed forecasting for wind farm based on least squares support vector machine (LS-SVM) with the atmospheric pressure and temperature as the input. Meanwhile, the grid search was used to determine the parameters of the LS-SVM model. The obtained results of this proposed method are basically in accordance with the values of actual wind speed. Ref. [18] used the least squares support vector machine (LS-SVM) and based on actual wind speed data measured in a certain wind farm to forecast the hour-ahead wind speed of this wind farm. The prediction result shows the mean average percentage error of the predicted wind speed is only $8.55 \%$. Ref. [19] used the support vector machine (SVM) technique to forecast the short-term wind speed. The forecasting result shows the mean average percentage error of the predicted wind speed is only $10.07 \%$. Ref. [20] proposed a new wind speed forecasting method which combines the GMDH neural network and fuzzy logic theory. This method fuzzy the neurons and introduced the feedback loop, which hybrid the Low-dimensional computing capacity of GMDH and the High-dimensional reasoning ability of fuzzy logic theory. The forecasting result shows this method can improve the forecasting accuracy. Ref. [21] proposed a support vector machine (SVM) method based on wavelet analysis and similar data. The training samples were built by extracting the similar data from large amounts of data. By using the wavelet decomposition, the original wind speed data was decomposed into trend signal of low frequency band and random signal of high frequency band. Finally, different SVM speed forecasting models were trained respectively and combined to obtain forecasting results. The simulation experiment shows this method can improve the forecasting accuracy.

Ref. [22] used the pattern recognition technique to select the sample, and employed the adaptive-network-based fuzzy inference system (ANFIS) model to forecast the wind speed. The forecasting result on Maui Island, Hawaii shows this method has a certain practicality. Ref. [23] used the Artificial Neural Network (ANN) model to forecast the wind speed. The parameters of ANN were determined by repeated training and testing on wind speed. The short-term wind speed forecasting result shows ANN method can achieve better result compared to that of ARMA model, but the treatment capability on mutation information is still limited. A wind power prediction system based on artificial neural network was established in Ref. [24]. The system relies on the numerical weather prediction, has friendly man-machine interface, and realizes seamless connection to the energy management system (EMS). The wind speed forecasting results indicate the prediction system is reliable and the root of mean square error (RMSE) is about 15\%. Ref. [25] proposed a LSSVM (Least squares support vector machines)-based wind speed forecasting model. The historical wind speed, pressure and temperature were select as the input variables, and the grid search method was employed to determine the optimal parameters of LSSVM model. The forecasting result shows this method has a good forecasting performance. Ref. [26] presented a multivariate local predictor for short-term wind speed prediction of wind farm. It sifted multivariate time series by correlation principle to reconstruct multivariate phase space, and searched the neighborhood of the prediction state points to build the support vector regression models. The calculation results show this proposed method can improve the searching efficiency of local predictor that can find much more similar neighbor points and also can effectively improve the accuracy of short-term wind speed prediction. 


\section{Hybrid Forecasting Method}

In general, the hybrid forecasting model refers to the combination of different approaches for wind speed forecasting, such as mixing statistical and soft computing approaches or combining SVM and PSO (Particle swarm optimization) method, etc.

Ref. [27] hybrid the time series technique and neural network to forecast the short-term wind speed. The time series model was used to select the input variables and multi-layer back propagation neural network and generalized regression neural network were used to conduct forecasting. The $10 \mathrm{~min}, 20 \mathrm{~min}$ and $30 \mathrm{~min}$ wind speed forecasting results show this method possesses higher accuracy. Ref. [28] proposed a short-term wind speed forecasting method based on wavelet decomposition (WD) and least square support vector machine (LSSVM). The verification results of wind farms in Hong Kong region and the Hexi Corridor in Northwest China show that the proposed method can improve the accuracy of one-step ahead wind speed forecasting. Based on similarity curves, Ref. [29] proposed a back propagation (BP) neural network wind speed forecast method to improve the accuracy of wind speed forecast by using the seasonal cycle fluctuation of wind speed. Combining with time series analysis and grey forecasting method, a wind speed forecasting method was performed. Simulation results demonstrate that the proposed methodology can improve the accuracy of wind speed forecast. Ref. [30] combined the genetic algorithm and back propagation (BP) neural network to propose a new wind speed forecasting model. The wind speed, intensity, humidity, and barometric pressure were taken as the input of BP neural network model, and the genetic algorithm was used for determine the initial weights and thresholds. The $1 \mathrm{~h}$-ahead, $2 \mathrm{~h}$-ahead and $3 \mathrm{~h}$-ahead of wind speed were forecasted, and the results show this method is effective.

Ref. [31] proposed a new wind speed prediction scheme that uses rough set method. The key factors that affect the wind speed prediction were identified by rough set theory. Then the rough set neural network prediction model was built by adding the key factors as the additional inputs to the pure chaos neural network model. The forecasting result on a wind farm of Heilongjiang province show that the prediction accuracy of this proposed method is the best compared to that of chaos neural network model and persistence model. Ref. [32] proposed a hybrid wind speed forecasting model based on the wavelet decomposition, differential evolutionary and support vector machine. Compared to that of Cross-validation support vector machine method and BP neural network model, this proposed method has higher wind speed forecasting accuracy. Ref. [33] proposed a time series ANN (artificial neural network) method for wind speed forecasting. In the proposed method, the mathematical model was built by time series method to obtain the basic parameters of wind speed characteristics, then these parameters was used to choose input variables of ANN. Meanwhile, a rolling method to adjust weight factors was put forward. The forecasting result shows this method can effectively improve the wind speed forecasting accuracy. Ref. [34] built the wind speed forecasting model based on least squares support vector machine theory, and tried to use Ant Colony Algorithm theory to optimization choice for parameters. Using the wind farm observed wind speed (sampling interval is 30 minutes) of the day before four days to forecast the 48ind wind speed of the fifth day through this proposed wind forecasting model, and the MAPE is only $9.53 \%$. A wind speed forecasting model for wind farm based on wavelet decomposition, support vector machine and genetic algorithm is proposed in Ref. [35]. Through wavelet decomposition, the data were preprocessed and through genetic algorithm, the parameters were optimized. The simulation results show that the forecast wind speed is following the true value, what's more, the model can adapt to different wind data.

The spatial translation method was presented to make the data collection easier by reducing the number of correlative sites, which used RBF (Radial Basis Function) neural network to set the nonlinear relation of wind speed between correlative sites in Ref. [36]. A multi - interval forecast model based on the spatial correlation was built, which divided the forecast period into several intervals, analyzed the chronological correlation of wind speed between wind farm and correlative site and selected the best correlative site for each interval. The example calculation shows that the wind speed forecast accuracy is improved and the training time is reduced. A forecasting method based on LS-SVM considering the factors related is proposed to choose factors having significant impacts on the wind speed through correlation analysis as the reference quantity for information characteristics of the wind speed, to adopt grey relation analysis to pretreat the speed data, and search the historical speed with highly similar features to the forecasting day as the training samples of the LS-SVM model in Ref. [37]. The actual examples prove that the wind speed forecasting accuracy and reliability of this method are effectively improved. A short-term combination forecasting model of wind speed was developed based on the seasonal periodicity and time-continuity of wind energy in Ref. [38]. With the pattern recognition technique, two kinds of samples to seasonal periodicity and time-continuity were selected separately. The two kinds of samples were used respectively to train two back propagation (BP) neural network models to obtain the lateral and vertical wind speed forecasting values. By importing the two values to a BP neural network again, the wind speed value was finally predicted. Ref. [39] proposes a least squares support vector machine (LSSVM) model optimized by the particle swarm optimization (PSO).The phase space of the chaotic wind speed time series was reconstructed by calculating the embedding dimension and the delay time of the wind speed time series. The PSO was used to optimize the parameters of the LSSVM. Then the improved LSSVM model can be used to forecast the wind speed. The results show the improved LSSVM can meet the accuracy requirements, and has a better forecasting 
performance than SVM prediction model and the BP neural network prediction model. A wind speed forecasting method based on wavelet-neural network and is proposed n Ref. [40]. In the proposed method, the original waveform was decomposed in different scales by wavelet function and the decomposed periodic components were forecasted by time series, and the rest parts were forecasted by neural network, finally the signal series were reconstructed to obtain complete wind speed forecasting result. Adding differential evolution algorithm, the convergence speed of the proposed method was improved and the local minimum problem was also solved.

Ref. [41] used SVM and genetic algorithm to forecast the wind speed. The genetic algorithm was employed to optimize the penalty factor $C$ and kernel parameter $\sigma^{2}$ of support vector machines. Compared with the general regression neural network(GRNN)method, this proposed forecasting method achieves better generalization ability and its average absolute value of relative error is only $8.32 \%$. A hybrid method based on empirical mode decomposition (EMD) and time-series analysis was presented in Ref. [42]. The original wind speed sequences were firstly pretreated by EMD and decomposed into some intrinsic mode functions (IMF) and a residue. Then, according to their respective variation rule, each partition was modeled and forecasted using time-series analysis. Ultimately, all the forecasted values corresponded to these partitions were superposed to get the forecasted wind speed. The results indicate that the wind speed forecasting precision is improved. Ref. [43] used the wavelet package transform and support vector regression(SVR) to forecast the one hour to six hour-ahead wind speed forecasting of each ten minute. Firstly, in view of the non-stationary and nonlinear features of wind speed, the original wind speed sequence was decomposed into a series of sub-sequences; then these sub-sequences were respectively forecasted by SVR; finally, respective outputs were superposed to obtain final forecasted wind speed. The Adaboost algorithm was led in to improve back propagation (BP) neural network algorithm, and an Adaboost-based BP neural network method was proposed and applied to short-term wind speed forecasting in Ref. [44]. Results show using the proposed Adaboost-based BP neural network the accuracy of one or two hour-ahead wind speed forecasting was superior to respective forecasting accuracy by neural network and ARMA time series analysis, and the mean absolute percentage error of wind speed forecasting by the proposed algorithm was lower than $7.5 \%$ in high wind speed period (higher than $10 \mathrm{~m} / \mathrm{s}$ ).

A new approach for ultra-short-term wind speed forecasting is presented based on the phase space reconstruction technique and the local prediction method which can search the neighbors in the optimal neighborhood in the phase space and to build support vector regression (SVR) models in Ref. [45]. This approach finds the optimum neighborhood by considering of proportion of false neighbors, which guarantees the high similarity between neighbors and prediction state points, and the SVR model has a good capability of nonlinear fitness. A wind speed prediction model (WD-SVM) that uses data mining techniques of wavelet analysis and support vector machine, was developed in Ref. [46]. In this model, a given wind speed time series were decomposed by wavelet analysis into various layers that were predicted with support vector machines, and then by reconstructing the predicted values of each layer a prediction of wind speed was obtained. The average root-mean-square error of 10-minute average wind speed four hours in advance is $11.71 \%$. Ref. [47] forecasted the wind speed for $1 \mathrm{~h}$ ahead at a resolution of every $10 \mathrm{~min}$ by means of mathematical morphology and support vector regression. The original wind speed sequences were decomposed into a series of subsequences with different frequencies and wave characters by adaptive multi-scale morphological algorithm. Then, the subsequences with the method of SVR were predicted respectively. Finally, the final predicted wind speed was calculated by the superposition of respective predictions. An interval type-2 fuzzy logic model was proposed to forecast short-term wind speed time series based on the singular value decompose and back-propagation (SVD-BP) hybrid iterative arithmetic in Ref. [48]. The BP arithmetic was used to tune input, antecedent and consequent parameters, the SVD method was used to choose reasonable rules. For testing the performance, both a type-1 fuzzy logic model and an interval type-2 fuzzy logic model using only BP arithmetic were designed as comparable benchmarks. The simulation results show that the interval type-2 fuzzy logic model using the SVD-BP hybrid iterative arithmetic has the best performance and practicability.

A wind power prediction method based on empirical mode decomposition (EMD) and support vector machine (SVM) is proposed in Ref. [49]. The wind speed data was firstly decomposed into a series of components with stationary by using EMD to reduce the influence between different feature information. Then, different models were built and different kernel functions and parameters were chosen to deal with each group of data by using SVM in order to improve the forecasting accuracy. Finally, short term wind power forecasting was made based on wind speed data through a practical wind power curve. A combined short-term wind speed forecasting model based on D-S evidence theory was proposed in Ref. [50]. The forecasting models of time series, BP neural network and support vector machine wer adopted to respectively forecast the wind speed. Based on the analysis of forecast errors, D-S evidence theory was applied to fuse these three models. The wind speed data for several days before were taken as the fusion samples to calculate the corresponding basic trust distribution functions, which were then fused. The results of fusion were taken as the weights of the wind speed forecasting model and the wind speed of the day to be forecasted was calculated. Simulative results show that, the proposed combined forecasting model has smaller forecasting error and better effect. 


\section{Other Forecasting Methods}

Except the above wind power forecasting techniques, there are still some other forecasting methods developed by Chinese researchers to forecast the wind speed.

The short term wind speed prediction based on the Physical principles was performed in Ref. [51]. This approach employed the wind speed from the numerical weather prediction-NWP as an input data, a roughness change model and orographical change model were used to model the local effects of a wind farm. The predicted wind speed was compared with the measured wind speed under the typical wind conditions. The results show that the prediction value can basically meet the requirements of prediction precision. A combined model to predict wind speed based on improved fuzzy analytic hierarchy process (AHP) was presented in Ref. [52]. Taking predictive cycle of wind speed, the fluctuation of wind speed and the reliability of predictor to the predictive model as objectives, by means of fuzzy judgment matrix the optimal weight of the combined model was determined. The calculation results show that the prediction results by the proposed predictive model are more accurate than those from traditional single predictive model. The phase space reconstruction technology was used for short-term prediction of wind speed in Ref. [53]. Based on the fundamental relation of the delay time window $\Gamma$ and $m$ 、 $\tau$, several sets of optimal combinations of $m$ and $\tau$ were advanced, and the optimal combination was found. An effective method was presented to choose reference point, which determined near phase point by Euclidean distance and correlation degree among phase point. Some false neighboring points were kicked off to improve forecasting accuracy. On the selection of forecasting model, one-order local forecasting method and BP neural network model were used.

Based on grey predictor models, Ref. [54] presented one-step to four-step average ten-minute wind speed forecasting and give the residual error for steady wind. Wind speed predictions for unsteady wind and gust were also made. Then, taking the steady wind speed for instance, fitting parameters in various models that wind power changes with wind speed were obtained by modeling the relationship between real wind power and sequential wind speed. In order to enhance the forecasting precision of wind power, the function model fitting for wind power characteristic was established through comparing the precision of different models from the aspect of piecewise function and overall model. The forecasting result shows this method is effective. Ref. [55] proposed a chaos-based wind speed forecasting method. On the basis of applying phase space reconstruction to wind speed time series, the wind speed was forecasted by chaotic weighted zero-order local forecasting method; and then to remedy the insufficiency of above-mentioned method, namely the forecasting was carried out by searching near phase point under high embedded dimensions, a modified weighted zero-order local forecasting method, which determined near phase point by correlation degree among phase points, was proposed and a new approach to compute weighted coefficient was put forward to improve forecasting accuracy. Chaos theory and methods were used to solve the wind speed prediction problem in Ref. [56]. Firstly, the time delay and the embedding dimension were calculated by correlation integral approach for reconstructing phase space of wind speed time series. Then, wind speed chaotic prediction model of optimal neighborhood was proposed which gives overall consideration to the nearest neighbors' weights and generalized degrees of freedom, also an improved criterion for selecting optimal neighborhood. The practical calculation shows that the proposed model has superior predictive capability under the appropriate model parameters.

\section{Conclusions}

China's wind power has developed rapidly in the past few years, and ranked first in terms of cumulative installed capacity in the world since 2010 . Due to the intermittent and fluctuant characteristics, the wind power penetration will bring big impact on power systems, and accurately forecasting wind power generation can alleviate the negative effects. To fill the wind speed forecasting review gap, this paper reviews the current wind speed forecasting techniques in China from four aspects, which are statistical method, soft computing method, hybrid forecasting method and other forecasting methods. The articles written in Chinese which has been published in high-quality peer-reviewed Chinese journals are analyzed in details. The hybrid wind speed forecasting method which combines different methods may have a better forecasting performance in the field of wind speed forecasting, which has caused widespread concerns of China's researchers.

\section{Acknowledgements}

The authors thank the editor and reviewers for their comments and suggestions.

\section{References}

[1] 2014 Wind power development report published by GWEC. http://www.gwec.net/wp-content/uploads/2014/04/\%E5\%85\% A $8 \% \mathrm{E} 7 \% 90 \% 83 \% \mathrm{E} 9 \% \mathrm{A3} \% 8 \mathrm{E} \% \mathrm{E} 7 \% 94 \% \mathrm{~B} 5 \% \mathrm{E} 7 \% \mathrm{BB} \% 9 \mathrm{~F}$ \%E8\%AE\%A1\%E6\%95\%B0\%E6\%8D\%AE2013.pdf

[2] Zhao H, Guo S, Fu L. Review on the costs and benefits of renewable energy power subsidy in China [J]. Renewable and Sustainable Energy Reviews, 2014, 37: 538-549.

[3] http://www.cwpc.cn/cwpp/cn/services/cwpc-news-service/1-2 $020 /$

[4] Tascikaraoglu A, Uzunoglu M. A review of combined approaches for prediction of short-term wind speed and power [J]. Renewable and Sustainable Energy Reviews, 2014, 34: 243-254. 
[5] Soman, Saurabh S., Hamidreza Zareipour, Om Malik. A review of wind power and wind speed forecasting methods with different time horizons. North American Power Symposium (NAPS), 2010. IEEE, 2010.

[6] Foley A M, Leahy P G, Marvuglia A, et al. Current methods and advances in forecasting of wind power generation [J]. Renewable Energy, 2012, 37(1): 1-8.

[7] DING Ming, ZHANG Lijun, WU Yichun. Wind speed forecast model for wind farms based on time series analysis [J]. Electric Power Automation Equipment, 2005, 25(8): 32-34.

[8] PAN Di-fu, LIU Hui, LI Yan-fei. A wind speed forecasting optimization model for wind farms based on time series analysis and kalman filter algorithm [J]. Power System Technology, 2008, 32(7): 82-86.

[9] Zhang Yanning, Kang Longyun, Zhou Shiqiong, et al. Wavelet analysis applied to wind speed prediction in predicate control system of wind turbine [J]. Acta Energiae Solaris Sinica, 2008, 29(5): 520-524.

[10] WANG Yao-nan, SUN Chun-shun, LI Xin-ran. Short-term wind speed simulation corrected with field measured wind speed [J]. Proceedings of the CSEE, 2008, 28(11): 94-100

[11] WANG Xiao-lan, LI Hui. Effective wind speed forecasting in annual prediction of output power for wind farm [J]. Proceedings of the CSEE, 2010, 30(8): 117-122.

[12] SUN Chun-shun, WANG Yao-nan, LI Xin-ran. A vector autoregression model of hourly wind speed and its applications in hourly wind speed forecasting [J]. Proceedings of the CSEE, 2008, 28(14): 112-117

[13] JIANG Jin-liang, LIN Guang-ming. Automatic station wind speed forecasting based on ARIMA model [J]. Control Theory \& Applications, 2008, 25(2): 374-376.

[14] FANG Jiang-xiao, ZHOU Hui, HUANG Mei, T.S. Sidhu. Short-term wind power prediction based on statistical clustering analysis [J]. Power System Protection and Control, 2011, 39(11): 67-73.

[15] YANG Xi-yun, SUN Han-mo. Wind speed prediction in wind farms based on time series model [J]. Journal of Chinese Society of Power Engineering, 2011, 31(3): 203-208.

[16] Liu Xingjie, Mi Zengqiang, Yang Qixun, et al. A novel multi-step prediction for wind speed based on EMD [J]. Acta Energiae Solaris Sinica, 2010, 25(4): 165-170.

[17] DU Ying, LU Ji-ping, LI Qing, et al. Short-Term wind speed forecasting of wind farm based on least square-support vector machine [J]. Power System Technology, 2008, 32(15): 62-66.

[18] ZENG Jie, ZHANG Hua. A wind speed forecasting model based on least squares support vector machine [J]. Power System Technology, 2009, 33(18): 144-147.

[19] Zhang Hua, Zeng Jie. Wind speed forecasting model study based on support vector machine [J]. Acta Energiae Solaris Sinica, 2010, 31(7): 928-932.

[20] WU Dong-liang, WANG Yang, GUO Chuang-xin, et al. Short-term wind speed forecasting in wind farm based on improved GMDH network [J]. Power System Protection and Control, 2011, 39(2): 88-93.

[21] YANG Xiyun, SUN Baojun, ZHANG Xinfang, et al. Short-term wind speed forecasting based on support vector machine with similar data [J]. Proceedings of the CSEE, 2012, 32(4): 35-41.

[22] WU Xing-hua, ZHOU Hui, HUANG Mei. Wind speed and generated power forecasting based on pattern recognition in wind farm [J]. Power System Protection and Control, 2008, 36(1): 27-32.

[23] Huang Xiaohua, Li Deyuan, Lv Wenge, et al. Wind speed forecasting with artificial neural networks model [J]. Acta Energiae Solaris Sinica, 2011, 32(2): 193-197.

[24] FAN Gao-feng, WANG Wei-sheng, LIU Chun. Artificial neural network based wind power short term prediction system $[\mathrm{J}]$. Power System Technology, 2008, 32(22): 72-76.

[25] DU Ying, LU Ji-ping, LI Qing, et al. Short-Term wind speed forecasting of wind farm based on least square-support vector machine [J]. Power System Technology, 2008, 32(15): 61-66.

[26] GUO Chuangxin, WANG Yang, SHEN Yong, et al. Multivariate local prediction method for short-term wind speed of wind farm [J]. Proceedings of the CSEE, 2012, 32(1): 24-31.

[27] CAI Kai, TAN Lun-nong, LI Chun-lin, et al. Short-Term wind speed forecasting combing time series and neural network method [J]. Power System Technology, 2008, 32(8): 82-85.

[28] WANG Xiao-lan, WANG Ming-wei. Short-Term wind speed forecasting based on wavelet decomposition and least square support vector machine [J]. Power System Technology, 2010, 34(1): 179-184.

[29] ZHANG Guoqiang, ZHANG Boming. Wind speed and wind turbine output forecast based on combination method [J]. Automation of Electric Power Systems, 2009 (18): 92-95.

[30] WANG De-ming, WANG Li, ZHANG Guang-ming. Short-term wind speed forecast model for wind farms based on genetic BP neural network [J]. Journal of Zhejiang University (Engineering Science), 2012, 46(5): 837-841.

[31] GAO Shuang, DONG Lei, GAO Yang, et al. Mid-long term wind speed prediction based on rough set theory $[\mathrm{J}]$. Proceedings of the CSEE, 2012, 32(1): 32-37.

[32] PENG Chunhua, LIU Gang, SUN Huijuan. Wind speed forecasting based on wavelet decomposition and differential evolution-support vector machine for wind farms [J]. Electric Power Automation Equipment, 2012, 32(1): 9-13.

[33] YANG Xiu-yuan, XIAO Yang, CHEN Shu-yong. Wind speed and generated power forecasting in wind farm [J]. Proceedings of the CSEE, 2005, 25(11): 1-5.

[34] Zeng Jie, Zhang Hua. Wind speed forecasting model study based on least squares support vector machine and ant colony optimization [J]. Acta Energiae Solaris Sinica, 2011, 32(3): 296-300.

[35] Luo Wen, Wang Lina. Short-Term wind speed forecasting for wind farm [J]. Transactions of China Electrotechnical Society, 2011, 26(7): 68-74.

[36] LI Wenliang, WEI Zhinong, SUN Guoqiang, et al. Multi-interval wind speed forecast model based on improved spatial correlation and RBF neural network [J]. Electric Power Automation Equipment, 2009 (6): 89-92.

[37] LI Ran, CHEN Qian, XU Hong-rui. Wind speed forecasting method based on LS-SVM considering the related factors [J]. Power System Protection and Control, 2010 (21): 146-151. 
[38] JIANG Xiaoliang, JIANG Chuanwen, PENG Minghong, et al. A short-term combination wind speed forecasting method considering seasonal periodicity and time-continuity $[\mathrm{J}]$. Automation of Electric Power Systems, 2010 (15): 75-79.

[39] SUN Bin, YAO Hai-tao. The short-term wind speed forecast analysis based on the PSO-LSSVM predict model [J]. Power System Protection and Control, 2012, 40(5): 85-89.

[40] YANG Qi, ZHANG Jianha, Wang Xiangfeng, et al. Wind speed and wind power generation forecast based on Wavelet - Neural Networks model [J]. Power System Technology, 2009, 33(17): 44-48.

[41] YANG Hong, GU Shi-fu, CUI Ming-dong, et al. Forecast of short-term wind speed in wind farms based on GA optimized LS-SVM [J]. Power System Protection and Control, 2011, 39(11): 44-48.

[42] Liu Xingjie, Mi Zengqiang, Yang Qixun, et al. Wind speed forecasting based on EMD and time-series analysis [J]. Acta Energiae Solaris Sinica, 2010, 31(8): 1037-1041.

[43] CHEN Pan, CHEN Haoyong, YE Rong, et al. Wind speed forecasting based on combination of wavelet packet analysis with support vector regression [J]. Power System Technology, 2011, 35(5): 177-182.

[44] WU Junli, ZHANG Buhan, WANG Kui. Application of Adaboost-based BP neural network for short-term wind speed forecast [J]. Power System Technology, 2012, 36(9): 221-225.

[45] WANG Yang, ZHANG Jinjiang, WEN Bojian, et al. An optimal neighborhood in phase space based local prediction method for ultra-short-term wind speed forecasting [J]. Automation of Electric Power Systems, 2012, 35(24): 39-43.

[46] ZHANG Hua, YU Yongjing, FENG Zhijun, et al. Wind speed forecasting model based on wavelet decomposition and support vector machine [J]. Journal of Hydroelectric Engineering, 2012, 31(1): 208-212.
[47] CHEN Pan, CHEN Hao-yong, YE Rong. Wind speed forecasting based on multi-scale morphological analysis [J]. Power System Protection and Control, 2010 (21): 12-18.

[48] Zheng Gao, Xiao Jian, Wang Jing, et al. Forecasting study of short-term wind speed based on interval type-2 fuzzy logic method [J]. Acta Energiae Solaris Sinica, 2012, 32(12): 1792-1797.

[49] YE Lin, LIU Peng. Combined Model Based on EMD-SVM for Short-term Wind Power Prediction [J]. Proceedings of the CSEE, 2011, 31(31): 102-108.

[50] Liu Yanan, Wei Zhinong, Zhu Yan, et al. Short-term wind speed forecast based on D-S evidence theory [J]. Electric Power Automation Equipment, 2013, 33(8): 131-136.

[51] Feng Shuanglei, Wang Weisheng, Liu Chun, et al. Short term wind speed prediction based on physical principle [J]. Acta Energiae Solaris Sinica, 2011, 32(5): 611-616.

[52] HUANG Wen-jie, FU Li, XIAO Sheng. A predictive model of wind speed based on improved fuzzy analytical hierarchy process [J]. Power System Technology, 2010, 34(7): 164-168.

[53] Lü Tao, TANG Wei, SUO Li. Prediction of short-term wind speed in wind farm based on chaotic phase space reconstruction theory [J]. Power System Protection and Control, 2010 (21): 113-117.

[54] LI Jun-fang, ZHANG Bu-han, XIE Guang-long, et al. Grey predictor models for wind speed-wind power prediction $[\mathrm{J}]$. Power System Protection and Control, 2010, 38(19): 151-159.

[55] LUO Hai-yang, LIU Tian-qi, LI Xing-yuan. Chaotic forecasting method of short-term wind speed in wind farm $[\mathrm{J}]$. Power System Technology, 2009, 33(9): 67-71.

[56] Ding Tao, Xiao Hongfei. Wind speed chaotic prediction model based on optimal neighborhood [J]. Acta Energiae Solaris Sinica, 2011, 32(4): 560-564. 